
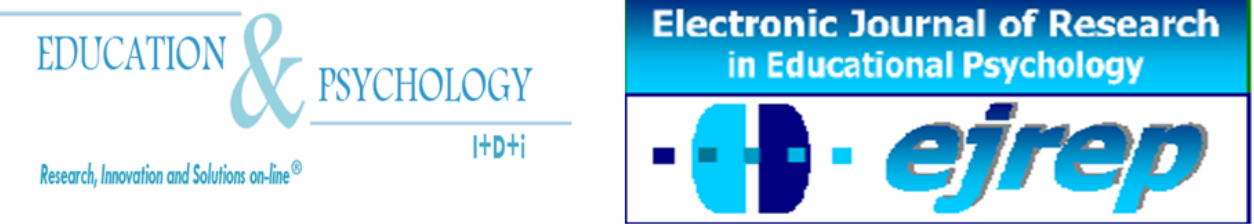

Editorial EOS

\title{
Las TIC en las titulaciones universitarias de grado: análisis del conocimiento y uso en el alumnado de la Universidad a Distancia
}

\section{Raquel Suriá}

Dpto. de Comunicación y Psicología Social, Universidad de Alicante

\section{España}

Correspondencia: Raquel Suriá. C/ Brasil, 10, $1^{\circ}$ B. Alicante. 03008. España. E-mail: raquel.suria@ua.es (C) Education \& Psychology I+D+i and Editorial EOS (Spain) 


\section{Resumen}

Introducción. En la actualidad, el uso de las TIC en el Espacio Europeo de Educación Superior se ha convertido en un tema estrella. Una de las universidades que mayor énfasis va a poner en su utilización es la universidad a distancia. Nuestro estudio examinó a un grupo de alumnos de un centro de la Universidad Nacional de Educación a Distancia para conocer el grado de conocimiento y utilización que hacen de las TIC así como de la necesidad de aprender a manejarlas para cursar las titulaciones de grado. Esto nos ayuda a identificar las diferentes necesidades requeridas por los alumnos con el fin de diseñar y proponer medidas proactivas.

Método. Participaron 178 estudiantes de la UNED del centro asociado de Elche. De ellos, 100 estaban matriculados en primero de Psicología y 78 en la titulación de Educación Social durante el curso 2009-2010. Se les administró un cuestionrio con 22 ítems para investigar la utilización y experiencia_con las TIC. Los datos se analizaron mediante una prueba de Chi Cuadrado de Pearson.

Resultados. Los resultados muestran diferencias estadisticamente significativas en el uso y conocimiento de las TIC, entre los grupos, observándose que la mayoría del alumnado no se sienten preparado para utilizar los recursos informáticos en sus estudios.

Discusión y conclusiones. Los resultados principales del presente trabajo indicaron que el alumnado que estudia en la universidad a distancia, no utiliza las TIC habitualmente, por lo que su aplicación al proceso de enseñanza y aprendizaje, no llega a alcanzar el mismo nivel de utilización que el logrado en las universidades tradicionales, al no recibir el alumnado a distancia, una formación que facilite su uso.

Palabras Clave: Nuevas tecnologías; Proceso de Bolonia; Educación Superior; Enseñanza a distancia; Innovación educativa.

Recibido: 27/05/10 Aceptación Inicial: 02/06/10 Aceptación Definitiva: 29/07/10 


\title{
ICT in undergraduate university programs: assessment of their use in distance education pupils
}

\begin{abstract}
Introduction. Currently, the discussion on the use of ICTs in higher education has turned into a key issue. One of the universities that stand out their use is the university at a distance. Our study examined a group of students at distance university center to know the degree of knowledge and use of ICT, as well as the need to learn how to handle them to pursue undergraduate degrees. This helps us to identify the different needs required by students in order to design and propose proactive measures.
\end{abstract}

Method. A total of 178 students from UNED participated in the study. 100 of these students, were enrolled in Psychology and 78 in Social Education for 2009-2010 academic year at the partner institution of the UNED from Elche. They were given a 22 items questionnaire to investigate the use and experience about ICTs (information and communication technologies). Data were analyzed by Chi square statistic.

Results. Results indicate statistically significant differences in the use and knowledge of ICTs between groups, showing that most students do not feel prepared to use technological resources in their studies.

Conclusion. In conclusion, the main results of the present study indicated that students studying at the distance university do not use ICT frequently. Due to this lack of training, its application to the teaching and learning process does not reach the same level as in traditional universities.

Keywords: Information and Communications Technologies; Bologna Process; Higher Education; Educational innovation.

Received: 05/25/10 Initial Acceptance: 06/02/10 Definitive Acceptance: 07/29/10 


\section{Introducción}

La firma del Tratado de Bolonia en 1999, ha supuesto una revolución en la enseñanza universitaria en España. En esta declaración se hace énfasis en la necesidad de promover la construcción de un "Espacio Europeo de Educación Superior", organizado conforme a ciertos principios (calidad, movilidad, diversidad, competitividad) y con ello, ciertos cambios que van más allá de la tradicional acumulación de conocimientos (Álvarez, 2003; Biggs, 2005; Bonsón y Benito, 2005; Klenowski, 2005; Ruiz, 2005; Velázquez y Martínez, 2004). En este sentido, además de las clases presenciales tradicionales, cobra especial relevancia la evaluación continua para favorecer el proceso de enseñanza aprendizaje. De esta forma, con el fin de implicar a los alumnos más activamente en el aprendizaje, el plan de Bolonia incide en la necesidad de la presencia del alumnado de forma habitual en el aula, se acentúa la importancia de la realización de actividades de manera continuada (Bain, 2006; Kaftan, Burke y Haack, 2006), a la vez que las Tecnologías de la Información y de la Comunicación juegan un papel clave, ofreciendo nuevos contextos y posibilidades para el desarrollo de estas competencias. Este aspecto que de forma general, favorece el aprendizaje, puede tener implicaciones importantes para el alumnado de algunas universidades en las que la asistencia continua es escasa o a veces inexistente.

Éste es el caso de las universidades a distancia, las cuales se caracterizan por la ausencia de la presencialidad del alumnado a las clases tradicionales ya que imparten sus estudios a distancia de acuerdo a un criterio bastante diferente al de las universidades presenciales. Así, aspectos como la flexibilidad en cuanto al manejo de los propios tiempos por parte del estudiante, la ausencia del requisito de asistencia periódica a clase y el alto grado de autonomía que los estudiantes tienen a la hora de seguir sus estudios, son aspectos característicos de estas universidades, que sin duda, animan a multitud de alumnos a cursar estudios superiores.

En este sentido, los recursos que ofrece el profesorado de estos centros con el objetivo de subsanar los problemas derivados de la no asistencia del alumnado no presencial, son las nuevas tecnologías como apoyo a la docencia. Es evidente que con la incorporación de tecnologías al ámbito educativo se potencian los modelos de educación a distancia ya existentes y la creación de nuevas propuestas con fines de desarrollo profesional y de formación permanente. Asimismo, facilitan los procesos educativos y comunicacionales que implican el acercamiento entre los agentes involucrados en la enseñanza aprendizaje (Delgado-García y Oli- 
ver-Cuello, 2007). De esta forma, mediante plataformas virtuales, los equipos docentes de las universidades a distancia ofrecen videoconferencias a los alumnos y éstos pueden acceder a tutorías virtuales así como a foros y chats para interaccionar con otros compañeros y con los tutores (Fernandez, 2000; Ortega e Isla-Montes, 2000).

No obstante, aunque es evidentemente necesaria la aplicación de las tecnologías de la información a la educación, podemos encontrarnos con algunas limitaciones que en principio, pueden alejar más a los alumnos que empiezan a cursar su primer año en las titulaciones de grado en estas universidades (Adell, 1998; McClintock, 1993). Así, un aspecto que caracteriza a los alumnos de la universidad a distancia es su perfil heterogéneo tanto por edad (hay estudiantes de todas las edades) como por perfil personal y profesional (desde trabajadores que buscan obtener una titulación adicional a amas de casa y personas con interés en retomar o iniciar estudios superiores). Es por ello que, con frecuencia, nos encontramos con un elevado porcentaje de alumnos que por sus ocupaciones, dispongan de poco tiempo para estudiar. Asimismo y, sobre todo, en los primeros cursos, otros estudiantes se incorporen a la universidad desprovistos de hábitos y técnicas de estudio, por lo que, el hecho de estudiar en sí, puede considerarse una meta difícil de poder llevarse a cabo.

$\mathrm{Si}$, además de estas condiciones, nos encontramos con que el uso y apoyo que los alumnos pueden encontrar en los medios tecnológicos para suplir la falta de clases presenciales puede ser escaso, debido en parte, a la inexistencia de recursos informáticos o al desconocimiento del manejo informático, vamos a encontrarnos que estas innovaciones tecnológicas pueden ser obstáculos que dificulten en principio, el avance en la universidad para un gran número de alumnos que inician sus titulaciones (Marquès, 2003).

En este sentido, aunque, si es cierto, que la mayoría de centros pertenecientes a la universidad a distancia ponen a disposición del alumnado los recursos tecnológicos necesarios para consultar a los profesores, no es éste el recurso más utilizado. Así, las vías más frecuentes para establecer contacto con los profesores, consultar dudas o entregar prácticas han sido el correo ordinario y las consultas telefónicas, no siendo hasta la instauración del nuevo plan de estudios obligatoria la utilización de las tecnologías. De este modo, es necesario que el alumno tenga unos mínimos conocimientos del uso de las tecnologías para acceder a los foros de las asignatutas, realizar las prácticas vía online y descargar los materiales de estudio en la web de los profesores. 
Ante esto, se nos plantean algunas cuestiones referidas a los alumnos que comienzan sus estudios en las universidades a distancia, así, ¿utilizan los estudiantes las nuevas tecnologías?, ¿tienen conocimiento sobre su manejo?, ¿disponen de acceso al material informático necesario para reforzar su enseñanza aprendizaje?, ¿son conscientes de la importancia de utilizar las TIC para cursar sus estudios de grado? Por último, ¿consideran los estudiantes que inician sus estudios, que es positivo realizar un cursillo orientador sobre el manejo de las nuevas tecnologías?

\section{Objetivos}

A partir de estas consideraciones previas los objetivos que pretendemos alcanzar con esta investigación se pueden concretar en los siguientes:

a) Conocer el grado de conocimiento, uso y opiniones en relación a la utilización de las TIC en las nuevas titulaciones de grado de los estudiantes que comienzan sus estudios en las universidades a distancia.

b) De manera complementaria, pretendemos averiguar el grado de acuerdo que tienen los alumnos en cuanto a la necesidad de que se incluyan cursos, seminarios o talleres para formarles previamente en el uso de las TIC.

\section{Método}

Participantes

La muestra de estudio se compuso por 178 alumnos (134 mujeres y 44 varones), de primer curso de grado de la Universidad Nacional de Educación a Distancia. De ellos, 78 se matricularon en Educación Social y los otros 100 alumnos estaban matriculados en Psicología durante el curso 2009-2010 en el centro asociado de Elche de la UNED. 


\section{Instrumentos}

Para realizar el estudio se utilizó como instrumento de recogida de datos un cuestionario diseñado ad hoc para alcanzar los objetivos del estudio. El cuestionario se compuso de 2 bloques, el $1^{\circ}$ destinado a examinar el perfil sociodemográfico de los estudiantes (6 ítems) y el $2^{\circ}$ bloque dedicado a explorar el uso, disponibilidad y actitudes que tienen los estudiantes ante las TIC. Este apartado, consta de 16 preguntas cerradas, con respuestas tipo si/no y Likert, con 5 alternativas de respuesta (desde $1=$ nada, hasta $5=$ mucho) y enfocado a varios aspectos, el $1^{\circ}$ de ellos dedicado a conocer la experiencia (4 ítems) y accesibilidad (6 ítems) a las nuevas tecnologías y el $2^{\circ}$ aspecto, destinado a averiguar la importancia y necesidad que los alumnos perciben sobre la adquisición en cuanto a la formación en las TIC para avanzar en sus estudios (6 ítems).

Para cumplir con los requisitos de validez de contenido se aplicó el protocolo de validación de contenido, que determina la relevancia o representatividad de los ítems en relación a la muestra establecida (Latiesa, 1996; Losada y López-Feal, 2003). Para ello, 3 jueces expertos respondieron a un cuestionario dicotómico que indagaba sobre la validez o no de cada ítem propuesto, a los resultados obtenidos se aplicó la Distribución Binomial para cada ítem, quedando el cuestionario compuesto por 22 ítems (ver Anexo). En relación a la validez operativa los mismos expertos efectuaron recomendaciones en función de las cuales se ajustaron las preguntas. La información requerida buscaba opiniones sobre la claridad de las instrucciones y preguntas efectuadas al estudiante, los términos empleados para éste grupo, secuencia y número de ítems. La fiabilidad del cuestionario se determinó mediante el Coeficiente Alfa de Crombach, obteniendo un índice de consistencia interna de 0.67.

\section{Procedimiento}

El procedimiento de recogida de información consistió en la aplicación del cuestionario a una muestra de alumnos de primer curso de algunas titulaciones impartidas en la UNED. El criterio de selección se basó en: 1) titulaciones en las que ya estuviera instaurado el nuevo plan; 2) titulaciones que tuvieran un elevado número de alumnos matriculados en el centro en el primer curso de esa titulación que acudan a alguna tutoría; 3) titulaciones en las que se desarrolla evaluación continua. Basándonos en esto, seleccionamos las titulaciones de Psicolog- 
ía y de Educación Social y tras explicar el objetivo del estudio a los alumnos, colaboraron de manera voluntaria y anónima al finalizar el primer parcial del curso académico 2009/10.

\section{Diseño y análisis estadístico}

Se ha realizado un análisis descriptivo de las frecuencias y los porcentajes válidos. Asimismo, se ha aplicado la prueba de Chi-cuadrado de Pearson para determinar la existencia de diferencias estadísticamente significativas entre las variables estudiadas en los distintos grupos de estudiantes en función de la titulación. El nivel de significación estadística en todo el estudio, para la aceptación o el rechazo de las hipótesis ha sido de $\mathrm{p}<.001$ y $\mathrm{p}<.05$. Elegimos esos niveles de significación con el objetivo de limitar la máxima probabilidad de error de tipo I, es decir, que la probabilidad de error en nuestras estimaciones fuera mínima.

\section{Resultados}

Respecto al perfil sociodemográfico de los alumnos participantes, en la Tabla I, describimos los datos relativos a la edad, sexo, estado civil y situación laboral.

Así, de los 178 alumnos participantes, el 75.3\%, $(n=134)$ de los cuestionarios corresponden a mujeres y el $24.7 \%(n=44)$ a varones, destacando la participación de las mujeres en ambas titulaciones. En lo referente a la edad, los alumnos participantes tienen edades comprendidas entre los 18 y los 44 años, destacando la participación del alumnado con el rango de 25 a 31 años con el $41.6 \%$. No obstante, si examinamos estos porcentajes según la titulación encontramos que el alumnado de Educación Social destaca en ese rango (22.5\%) mientras que los alumnos de Psicología destacan en el rango de 33 a $38(23.6 \%),\left(\chi_{(3,178)}^{2}=36.14, p<.001\right)$. En cuanto al estado civil, se observa que la mayoría de los participantes están solteros en ambas titulaciones (58.9\%), seguidos de los que están casados (39.4\%) y finalmente alrededor del $2 \%$ están separados.

En relación a su situación laboral, el 38.8\% de los alumnos participantes actualmente está trabajando, el $34.8 \%$ solo estudia, el $3.9 \%$ está incapacitado para trabajar y un $22.5 \%$ se dedica a las tareas del hogar. No obstante, al examinar la situación laboral según las titulaciones de nuestro estudio nos encontramos un mayor porcentaje de alumnos de Educación Social 
que se dedican exclusivamente a estudiar (28.1\%), seguidos de los que compaginan sus estudios con el trabajo (8.4\%), mientras que al examinar el grupo de Psicología se observa un mayor porcentaje de alumnos que compaginan sus estudios con el trabajo (30.3\%), seguidos de los que se dedican a las tareas del hogar (16.3), $\left(\chi_{(3,178)}^{2}=52.8, \mathrm{p}<.001\right)$, (Tabla I).

Tabla 1. Perfil sociodemográfico

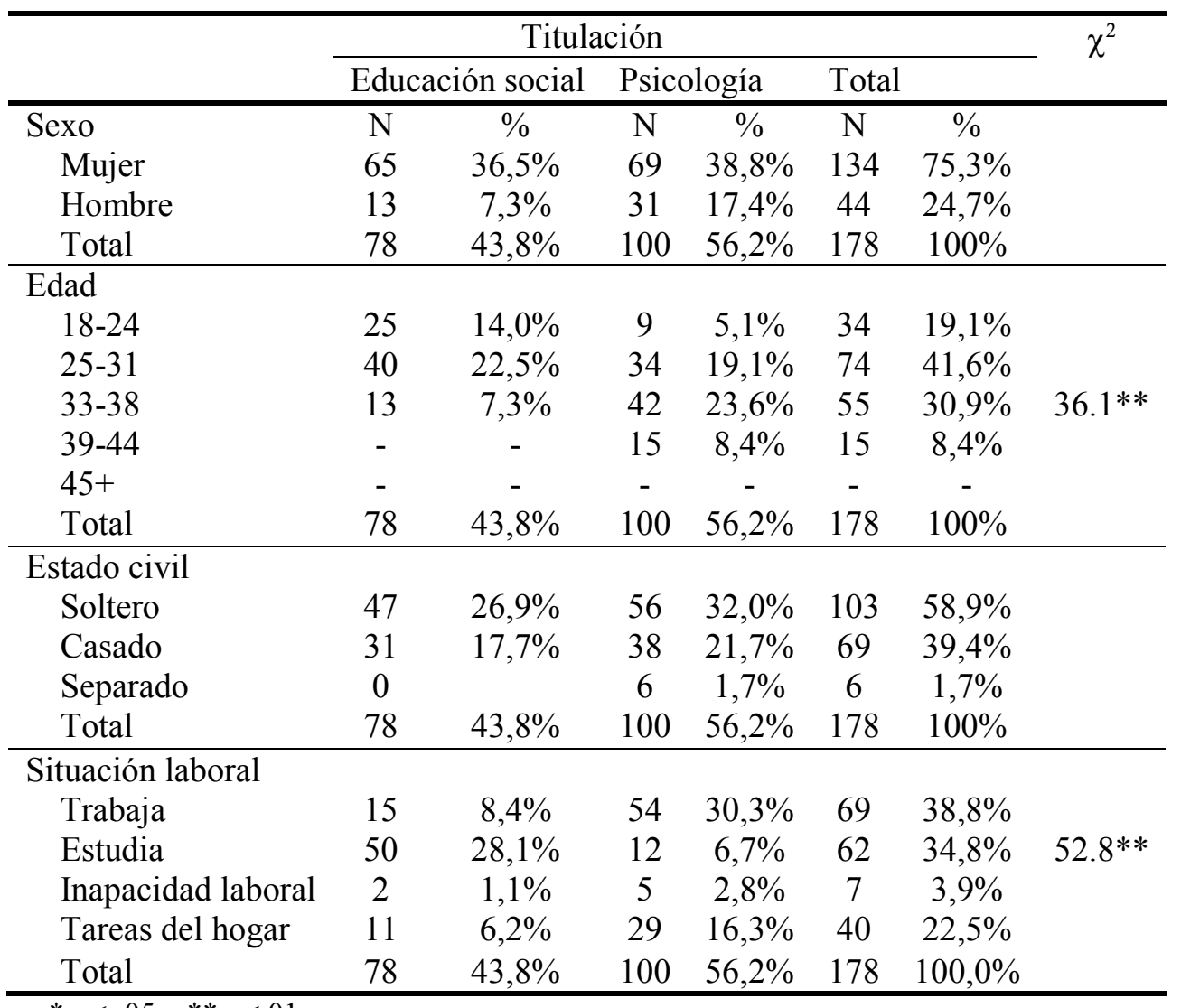

$* p<.05 \mathrm{y} * * p<.01$.

Con respecto al $2^{\circ}$ bloque del cuestionario concerniente a la accesibilidad, uso y manejo de las nuevas tecnologías observamos los siguientes resultados:

En referencia a la pregunta de si disponen de ordenador (Figura 1), encontramos que de los 178 alumnos, 96 de ellos no disponen de pc, mientras que 82 si tienen. Así, al examinar esta variable en función de la titulación, observamos un mayor porcentaje de alumnos de la carrera de Educación Social (55.1\%) que si tienen ordenador frente a los alumnos que cursan Psicología los cuales indicaron no disponer de éste $(61 \%),\left(\chi_{(1,178)}^{2}=4.58, \mathrm{p}<.05\right)$. 


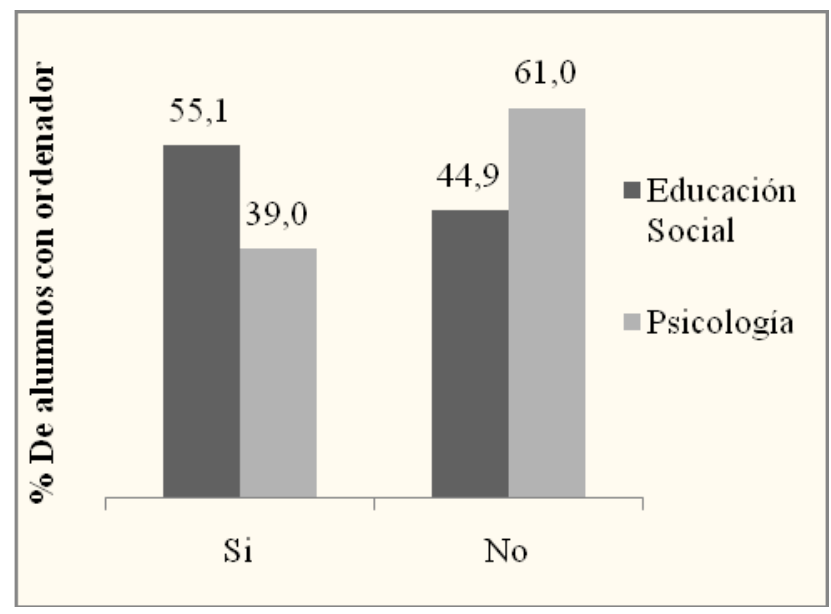

Figura 1. \% De alunmos con ordenador

En cuanto a la experiencia en el manejo de las TIC, es decir, el nivel de uso, observamos que la mayoría utiliza poco el ordenador (38.2\%), seguidos de los que no lo utilizan $(32 \%)$.

En relación a la pregunta de si tienen conexión a internet (Figura 2), encontramos que el $67.4 \%$ de estudiantes $(n=120)$ no tienen conexión, frente al $32.6 \%(n=58)$ de los alumnos que si tienen conexión, observándose que el porcentaje más alto de alumnos sin internet procedían de la titulación de Psicología (85\%), mientras que el porcentaje más elevado de alumnos con conexión a la red provenía del alumnado de Educación Social $(55.1 \%), \quad\left(\chi^{2}{ }_{(1,178)}\right.$ $=32.12, \mathrm{p}<.001)$.

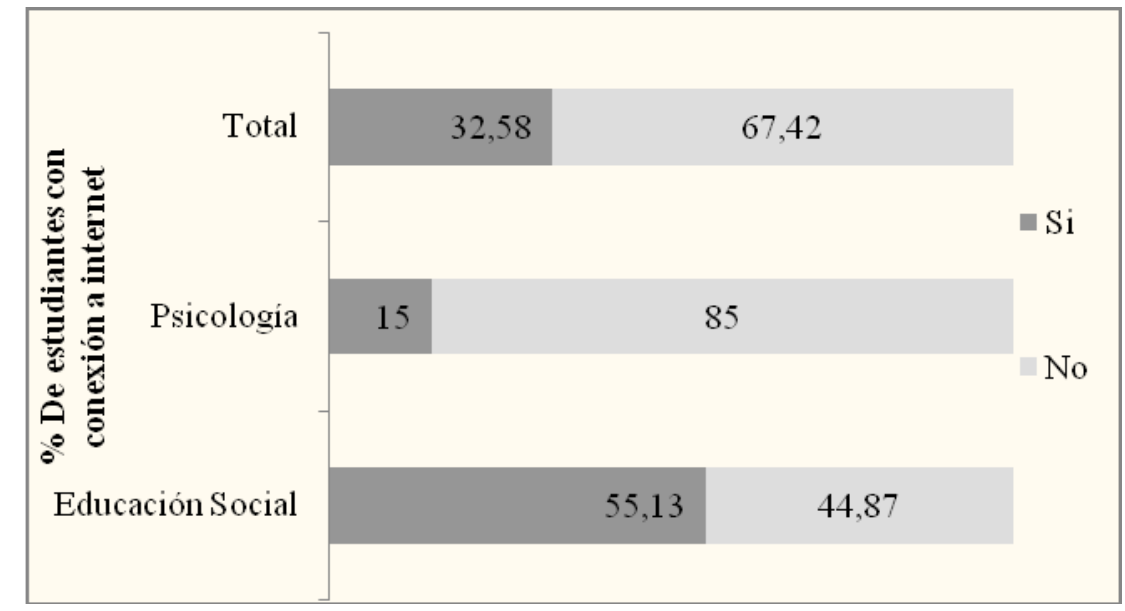

Figura 2. \% De estudiantes con conexión a internet 
En cuanto al nivel de conocimiento de las TIC, encontramos que el $37.1 \%$ de estudiantes sabe utilizar el ordenador algo, seguidos del 33.1\% que afirmó saber poco, un $18.5 \%$ indicó no tener ningún conocimiento sobre el manejo de las TIC y finalmente, el 11.2\% de alumnos afirmó saber bastante. Al examinar estos porcentajes según el grado o titulación, observamos que los porcentajes más altos en el grupo de alumnos de Educación Social se encontraron en saber utilizar algo el ordenador (50\%), mientras que en el grupo de Psicología destacó el rango de los que indicaron tener poco conocimiento de su uso $(43 \%),\left(\chi^{2}(3,178)\right.$ $=54.74, \mathrm{p}<.001)$

Los mismos resultados encontramos en cuanto al nivel de conocimiento para navegar por la red (Tabla 2), así, observamos que el 34.8\% de los alumnos participantes indicó saber navegar por internet algo, seguido de un $29.8 \%$ que afirmó saber poco, un $18.5 \%$ de estudiantes afirmó no saber nada sobre como navegar por internet y el 16.9\% afirmó saber bastante. Si examinamos estos porcentajes según la titulación, volvemos a observar porcentajes mayores en el grupo de Educación Social para el rango de saber navegar por la red algo (50\%), y en el grupo de Psicología que indicó saber navegar poco $(37 \%),\left(\chi_{(3,178)}^{2}=51.58, \mathrm{p}<.001\right)$.

Tabla 2. Experiencia en el uso de las TIC

\begin{tabular}{|c|c|c|c|c|c|c|c|c|c|}
\hline \multicolumn{10}{|c|}{ ¿Sabes manejar el ordenador? } \\
\hline & \multicolumn{2}{|c|}{ Nada } & \multicolumn{2}{|c|}{$\underline{\text { Poco }}$} & \multicolumn{2}{|c|}{$\underline{A \lg O}$} & \multicolumn{2}{|c|}{ Bastante } & $\chi^{2}$ \\
\hline Educación Social & 3 & $3.8 \%$ & 16 & $20.5 \%$ & 39 & $50.0 \%$ & 20 & $25.6 \%$ & \\
\hline Psicología & 30 & $30.0 \%$ & 43 & $43.0 \%$ & 27 & $27.0 \%$ & 0 & & $54.7 * *$ \\
\hline Total & 33 & $18.5 \%$ & 59 & $33.1 \%$ & 66 & $37.1 \%$ & 20 & $11.2 \%$ & \\
\hline \multicolumn{10}{|c|}{ ¿Sabes navegar por la Red? } \\
\hline Educación Social & 3 & $3.8 \%$ & 16 & $20.5 \%$ & 39 & $50.0 \%$ & 20 & $25.6 \%$ & \\
\hline Psicología & 30 & $30.0 \%$ & 37 & $37.0 \%$ & 23 & $23.0 \%$ & 10 & $10.0 \%$ & 51.8 \\
\hline \multirow[t]{3}{*}{ Total } & 33 & $18.5 \%$ & 53 & $29.8 \%$ & 62 & $34.8 \%$ & 30 & $16.9 \%$ & \\
\hline & \multicolumn{9}{|c|}{ ¿Cómo has aprendido a utilizar las TIC? } \\
\hline & \multicolumn{2}{|c|}{$\underline{\text { Yo solo }}$} & \multicolumn{2}{|c|}{ Compañeros } & \multicolumn{2}{|c|}{ Formación } & \multicolumn{2}{|c|}{$\frac{\text { No se usar- }}{\underline{\text { los }}}$} & \\
\hline Educación Social & 21 & $26.9 \%$ & 19 & $24.4 \%$ & 19 & $24.4 \%$ & 19 & $24.4 \%$ & \\
\hline Psicología & 30 & $30.0 \%$ & 6 & $6.0 \%$ & 27 & $27.0 \%$ & 37 & $37.0 \%$ & $13.0^{*}$ \\
\hline Total & 51 & $28.7 \%$ & 25 & $14.0 \%$ & 46 & $25.8 \%$ & 56 & $31.5 \%$ & \\
\hline \multicolumn{10}{|c|}{ ¿Qué recursos disponibles en la red utilizas? } \\
\hline & \multicolumn{2}{|r|}{ a } & \multicolumn{2}{|c|}{ Foros } & \multicolumn{3}{|c|}{$w w w$} & \multicolumn{2}{|c|}{ Ninguno } \\
\hline Educación Social & 28 & $35.9 \%$ & 5 & $6.4 \%$ & 15 & $19.2 \%$ & & 30 & $38.5 \%$ \\
\hline Psicología & 33 & $33 \%$ & 12 & $12 \%$ & 17 & $17 \%$ & & 38 & $38 \%$ \\
\hline Total & 61 & $34.3 \%$ & 17 & $9.6 \%$ & 32 & $18 \%$ & & 68 & $38.2 \%$ \\
\hline
\end{tabular}


En relación a como han aprendido a utilizar las TIC, observamos que un $31.5 \%$ de alumnos del estudio indicó no sabe utilizarlas, un 28.7\%, señaló que habían aprendido solos, el 25.8 que sabían utilizar las TIC por haber recibido formación previa y por último, el 14\% de estudiantes indicó que les habían enseñado otros compañeros. Al examinar estos porcentajes en función de la titulación, encontramos que el mayor porcentaje en Educación Social se encuentra entre los alumnos que aprendieron por su cuenta, mientras que el porcentaje más alto de alumnos de Psicoogía indicaron no saben utilizarlas, $\left(\chi_{(3,178)}^{2}=13.05, p<0.05\right)$.

Finalmente, para conocer cuáles son los recursos que con más frecuencia solían utilizar los estudiantes encuestados (Tabla 2), observamos que la mayoría de ellos indicaron no utilizar ningún recurso (38.2\%), seguidos de los que afirmaron utilizar el correo electrónico (34.3\%), las páginas web (18\%) y de los foros $(9.6 \%)$.

En referencia al bloque relacionado con el uso de las TIC y la titulación (Tabla 3), observamos que la mayoría de alumnos del estudio opinó que las nuevas tecnologías pueden ayudarles bastante a seguir a distancia su titulación (37.8), seguidos de los que indicaron que les ayudaría algo (37.1\%). Con respecto a la percepción sobre si es positiva la enseñanza virtual frente a la presencial encontramos que el 38.8\% de los alumnos afirmó que es bastante positivo, seguido del $38.2 \%$ que opinó que era algo positivo. Asimismo, al preguntarles su opinión sobre si es imprescindible saber manejar las nuevas tecnologías para cursar tus estudios, un $57.9 \%$ opinó que bastante, seguidos del $25.8 \%$ que indicó que algo.

En cuanto a la preparación de los alumnos para ayudarse de las nuevas tecnologías en su carrera observamos que la mayoría afirmaron sentirse poco preparados $(46.1 \%)$, seguidos del que los que indicaron no sentirse nada preparado (42.1\%). En referencia al grado en que los estudiantes creen que necesitan más formación en el manejo de las nuevas tecnologías para cursar sus estudios encontramos que el 58.4\% indicó necesitar bastante formación seguidos de los que indicaron necesitar mucho la formación en el manejo de las TIC (37.1\%). Por último, nos encontramos que el 58.4\% afirmó que estaría de acuerdo en recibir formación previa sobre el uso de las TIC para cursar su titulación, seguidos del 37.1\% que estaría muy de acuerdo. 
Tabla 3. Aplicación de tecnologías a titulación

\begin{tabular}{|c|c|c|c|c|c|c|c|c|c|c|}
\hline \multicolumn{11}{|c|}{ ¿Crees que las nuevas tecnologías pueden ayudarte a seguir a distancia tu carrera? } \\
\hline & \multicolumn{2}{|c|}{ Nada } & \multicolumn{2}{|c|}{ Poco } & \multicolumn{2}{|c|}{ Algo } & \multicolumn{2}{|c|}{ Bastante } & \multicolumn{2}{|c|}{ Mucho } \\
\hline Educación Social & 8 & $10.3 \%$ & - & - & 29 & $37.2 \%$ & 31 & $39.7 \%$ & 10 & $12.8 \%$ \\
\hline Psicología & 9 & $9.0 \%$ & 3 & $3.0 \%$ & 37 & $37.0 \%$ & 36 & $36.0 \%$ & 15 & $15.0 \%$ \\
\hline Total & 17 & $9.6 \%$ & 3 & $1.7 \%$ & 66 & $37.1 \%$ & 67 & $37.6 \%$ & 25 & $14.0 \%$ \\
\hline \multicolumn{11}{|c|}{ ¿Crees que es imprescindible saber manejar las nuevas tecnologías para cursar tus estudios? } \\
\hline Educación Social & - & - & - & - & 21 & $26.9 \%$ & 46 & $59.0 \%$ & 11 & $14.1 \%$ \\
\hline Psicología & - & - & - & - & 25 & $25.0 \%$ & 57 & $57.0 \%$ & 18 & $18.0 \%$ \\
\hline Total & - & - & - & - & 46 & $25.8 \%$ & 103 & $57.9 \%$ & 29 & $16.3 \%$ \\
\hline \multicolumn{11}{|c|}{ ¿En qué medida te sientes preparado para ayudarte de las nuevas tecnologías en tu carrera? } \\
\hline Educación Social & 33 & $42.3 \%$ & 35 & $44.9 \%$ & 9 & $11.5 \%$ & - & - & 1 & $1.3 \%$ \\
\hline Psicología & 42 & $42.0 \%$ & 47 & $47.0 \%$ & 9 & $9.0 \%$ & 1 & $1.0 \%$ & 1 & $1.0 \%$ \\
\hline Total & 75 & $42.1 \%$ & 82 & $46.1 \%$ & 18 & $10.1 \%$ & 1 & $0.6 \%$ & 2 & $1.1 \%$ \\
\hline \multicolumn{11}{|c|}{ ¿Crees que es positivo que se ofrezca la enseñanza virtual frente a la presencial? } \\
\hline Educación Social & 9 & $11.5 \%$ & 1 & $1.3 \%$ & 30 & $38.5 \%$ & 30 & $38.5 \%$ & 8 & $10.3 \%$ \\
\hline Psicología & 11 & $11.0 \%$ & 2 & $2.0 \%$ & 38 & $38.0 \%$ & 39 & $39.0 \%$ & 10 & $10.0 \%$ \\
\hline Total & 20 & $11.2 \%$ & 3 & $1.7 \%$ & 68 & $38.2 \%$ & 69 & $38.8 \%$ & 18 & $10.1 \%$ \\
\hline \multicolumn{11}{|c|}{ ¿En qué grado necesitas más formación en el manejo de las nuevas tecnologías para cursar tus estudios? } \\
\hline Educación Social & - & - & - & - & - & - & 42 & $53.8 \%$ & 36 & $46.2 \%$ \\
\hline Psicología & - & - & - & - & - & - & 52 & $52.0 \%$ & 48 & $48.0 \%$ \\
\hline Total & - & - & - & - & - & - & 94 & $52.8 \%$ & 84 & $47.2 \%$ \\
\hline \multicolumn{11}{|c|}{ ¿Estarías de acuerdo en recibir previa formación sobre el uso de las TIC para cursar tu titulación? } \\
\hline Educación Social & - & - & - & - & - & - & 46 & $59.0 \%$ & 32 & $41.0 \%$ \\
\hline Psicología & - & - & - & - & 8 & $8.0 \%$ & 58 & $58.0 \%$ & 34 & $34.0 \%$ \\
\hline Total & - & - & - & - & 8 & $4.5 \%$ & 104 & $58.4 \%$ & 66 & $37.1 \%$ \\
\hline
\end{tabular}

\section{Discusión y conclusiones}

En este trabajo se ha tratado de analizar la situación en cuanto al uso, experiencia y necesidad de formación en las TIC en los estudiantes que inician sus titulaciones de grado en el nuevo plan de estudios en la universidad a distancia.

Así, vemos que el perfil de los alumnos de nuestro estudio es bastante diferente al alumnado de las universidades tradicionales que inicia primer curso, ya que la mayoría de los alumnos participantes, se encuentra entre los 25 y 33 años, frente a los 18 y 25 años de los estudiantes de universidades presenciales (MICINN, 2009). Asimismo, un gran porcentaje de ellos ha formado una familia o tiene una situación laboral activa, perfil no reflejado en la mayoría del alumnado de las universidades tradicionales. 
Estos resultados coinciden con el perfil de estudiantes de otras universidades a distancia, en las que, como en líneas anteriores mencionamos, tienen un perfil bastante heterogéneo con respecto al alumnado estándar (González y Raposo, 2008; Sigalés, 2004).

Si analizamos estos resultados según la titulación, nos encontramos que las diferencias en función de esta variable, se incrementan según la titulación, alejándose más los alumnos de la carrera de Psicología del alumno universitario presencial. Una explicación razonable para este hecho puede consistir en la disponibilidad de la facultad de interés. Así, en el centro asociado de la universidad de nuestro estudio, que se encuentra situado en Elche, se imparten las titulaciones de Educación Social y de Psicología, sin embargo, existe la posibilidad de cursar la titulación de Psicología a través de una universidad presencial situada en esta misma localidad. Este hecho, puede llevar a que los alumnos más jóvenes prefieran o elijan la universidad tradicional y con ello, a que los alumnos que se matriculen en la universidad a distancia sean los de más edad. Por el contrario, la titulación de Educación Social, únicamente se imparte en la universidad de educación a distancia, y por tanto, a que el rango de edad del alumnado de esta titulación matriculado en el centro asociado de Elche de la universidad a distancia no sea solo de estudiantes que prefieren estudiar a distancia por sus circunstancias, sino que se le sumen los alumnos jóvenes que no puedan elegir una universidad presencial cercana a su lugar de residencia, incrementándose de este modo, alumnos de edades más tempranas.

En cuanto al uso de las TIC aplicadas a la educación a distancia no cabe duda de que cobran una especial relevancia en la interacción entre alumnos y entre estos, expertos y fuentes de información, aunque principalmente desde el nuevo plan de grados el interés se centra en el proceso facilitador de aprendizaje que ofrecen las TIC (Atienza, 2003; Blackstone, 2008; Bologna, 2008; Salinas, 2004).

Sin embargo, observamos que la experiencia en el manejo de las TIC en nuestra muestra de estudio, es decir, el nivel de accesibilidad y de uso, de la mayoría de alumnos es escaso y poco frecuente. Asi, al analizar el manejo y utilización del ordenador y de internet vemos que es muy diferente entre ambos grupos, destacando el alumnado de Educación Social por un mayor nivel de conocimiento y uso. Aunque dar una explicación a esto no es sencillo podríamos relacionar nuestros resultados con la disponibilidad de tiempo y edad de la muestra. Así, como en líneas anteriores hemos mencionado, el perfil de los alumnos de Psicología tiene

-1192- Electronic Journal of Research in Educational Psychology, 8(3), 1179-1200. 2010 ( $\left.\mathrm{n}^{\circ} 22\right)$. ISSN: 1696-2095. 
edades comprendidas entre 33 y 38 años, en situación laboral activa, además de un alto porcentaje dedicado a las tareas del hogar y casado. Estos resultados nos sugieren que el tiempo del que disponen deben compaginarlo con otras tareas, y por tanto, sea mucho más limitado que el de los alumnos con edades más jóvenes que pueden dedicar más tiempo a estudiar (Ruiz y Quintanal, 2008).

Asimismo, no podemos obviar que el rápido desarrollo de las nuevas tecnologías ha hecho posible que las nuevas generaciones de alumnos se adapten con mucha facilidad a las nuevas tecnologías (Coll, Rochera y Colomina, 2010). En este sentido, algunos autores (Lenhart, Rainie y Lewis, 2001; Oblinger y Oblinger, 2005; Veen and Vrakking, 2006) consideran que las generaciones que se han desarrollado a partir de los ochenta, han crecido en un contexto en el que las tecnologías digitales son parte consustancial de su vida diaria. En contrapartida, las generaciones de alumnos de mayor edad, los cuales no han incorporado las herramientas tecnológicas a su vida cotidiana de forma habitual encuentran limitaciones en cuanto a la adaptación a éstas (Area, 2000, 2001; Cabrero, 2004; García-Fernández y Bringué, 2002).

Esto puede desembocar en que los alumnos de más edad tengan en un principio, desventajas en comparación con los estudiantes más jóvenes a la hora de manejar el ordenador, navegar por la red, etc., debiendo distribuir su tiempo en aprender a manejar las TIC y en preparar adecuadamente sus asignaturas. Como apuntaban Touriñan, Rodríguez y Olveira (2003), la integración de las TICs como herramienta de trabajo diario puede constituir una desventaja para aquellos sectores que no tengan un fácil acceso y formación en cuanto a su utilización.

No obstante, nuestros resultados son alentadores con respecto a la aplicación de las TIC entre los alumnos que estudian a distancia las nuevas titulaciones de grado ya que, al analizar la predisposición que muestran a la hora de incorporar las tecnologías al proceso de enseñanza aprendizaje, observamos unos resultados muy positivos, considerando la mayoría de los alumnos participantes del estudio que es imprescindible aprender a utilizar las TIC, que les ayudaría bastante utilizarlas para su formación y que estarían dispuestos a recibir formación para poder realizar sus estudios exitosamente.

Es evidente que en la actualidad, el desarrollo de las TIC afecta prácticamente a todos los campos de nuestra sociedad y por supuesto, la educación no es una excepción. Así, y dado 
que las TIC facilitan la comunicación interpersonal y proporcionan un acceso inmediato a todo tipo de información, en la universidad, tanto profesores como estudiantes, debemos adaptarnos a estas herramientas, lo que implica un cambio metodológico de enseñanza que puede suponer enormes ventajas para todos. No obstante, aunque la implementación de las TIC está creando nuevos procesos de enseñanza aprendizaje, debemos ser conscientes de que existen algunas limitaciones que están incidiendo en la implementación de estas tecnologías a nivel universitario. Entre éstas y aún teniendo en cuenta la provisionalidad de los resultados de nuestro estudio, podemos mencionar que debido al perfil de alumnado que estudia en la universidad a distancia, la utilización de las TIC como instrumento habitual de aprendiaje, no puede alcanzar a un ritmo tan vertiginoso los objetivos que se pueden alcanzar en las universidades tradicionales ya que, al no estar acostumbrados a manejar con eficiencia las nuevas tecnologías, no podrán utilizar de forma óptima esta valiosa herramienta, viéndose de este modo, su proceso de aprendizaje afectado con consecuencias que limitarán el seguimiento y formación de los alumnos.

Asimismo, todo esto no solo limita al alumnado sino que, de algún modo, puede afectar a toda la comunidad universitaria. Así, no debemos olvidar que la difícil labor del profesorado, el cual, inmerso en las constantes adaptaciones a las que debe amoldarse y realizar (cambios en los planes de estudio, cursos de formación y reciclaje, adaptaciones curriculares para alumnos con NEE, etc.), se vea con la problemática de aplicar las tecnologías en la docencia que imparte a alumnos que no han adquirido el grado de conocimiento adecuado, influyendo esto en la calidad y motivación del docente para enseñar (Suriá, 2006).

Por ello, un importante eslabón por solventar en la incorporación de las TIC al nuevo plan de grados consiste en que la universidad posibilite o facilite cursos de formación que permita a los alumnos adquirir las habilidades necesarias para incorporar estas herramientas de manera cómoda, eficaz y crítica a su proceso de enseñanza aprendizaje.

\section{Limitaciones y futuras líneas de investigación}

En nuestro estudio nos hemos interesado en conocer el nivel de conocimiento y uso que tienen de las TICs los alumnos que estudian en las universidades a distancia con objeto de resolver posibles obstáculos entre los alumnos que optan estudiar en esta modalidad de estu- 
dio. Sin embargo, nuestro estudio presenta algunas limitaciones que es necesario tener en cuenta.

Así, esta investigación trabaja con los resultados obtenidos de un solo centro, el centro asociado de Elche de la Universidad de Educación a Distancia. Asimismo, se ha centrado en estudiantes de dos titulaciones, no podemos asegurar que los resultados se puedan generalizar a cualquier otro estudiante ubicado en una universidad a distancia distinta o que esté cursando otros estudios universitarios, por lo que nuestros resultados se limitan a la muestra de estudio.

Del mismo modo, nuestra muestra estuvo compuesta por los alumnos que con mayor o menor asiduidad asistían a tutorías en el periodo del estudio, no teniendo conocimiento de la opinión de los estudiantes que no asistieron a éstas. Por otra parte, es importante destacar que el presente estudio se encuentra limitado al análisis de asignaturas de primer curso de 20092010 y por lo tanto, la investigación no puede darse por concluida.

Así, esperamos en un futuro ampliar el estudio a más titulaciones a la vez que a otros centros asociados con el fin de obtener unos resultados más fiables. A su vez, sería interesante recoger y analizar los datos de varios cursos de manera que puedan ofrecer perspectivas longitudinal y transversalmente: la comparativa del mismo curso a través de toda la titulación así como de los diferentes cursos de cada una de ellas.

\section{Referencias}

Adell J. (1998). Redes y Educación. En J. De Pablos y J. Jiménez, (Eds.), Nuevas tecnologías, comunicación audiovisual y educación (pp.177-211). Barcelona: Cedecs.

Álvarez Méndez, JM. (2003). La evaluación a examen: ensayos críticos. Buenos Aires: Miño y Dávila.

Area, M. (2000). ¿Qué aporta Internet al cambio pedagógico en la educación superior? Quaderns Digitals. Consultado el 26/02/2009. En http:// www.ciberaula.net

Area, M. (2001). La alfabetización ante la cultura y la tecnología digital. La tensión entre mercado y democracia. En M. Area (Coord.), Educar en la sociedad de la información. Bilbao: Desclée de Brouwer.

Atienza, M. (2003). ¿Quién sabe qué es el e-learning? Educación, formación y trabajo, 69(1). Consultado el 10/09/2009. En: 
http://www.educaweb.com/esp/servicios/monografico/formacionvirtual/

Bain, K. (2006). Lo que hacen los mejores profesores universitarios. Valencia: Publicacions Universitat de València.

Biggs, J. (2005). Calidad del aprendizaje universitario. Madrid: Narcea.

Blackstone, T. (2008). Education and Training in the Europe of Knowledge. Consultado el 15/05/2009. En: http://www.uniroma3.it/downloads/297_Lezione\%20Blackstone.doc

Bologna Declaration (2008). Consultado el 11/03/2009. En: http://www.ond.vlaanderen.be/hogeronderwijs/bologna/documents/mdc/bologna_decl aration1.pdf

Bonsón, M. y Benito, A. (2005). Evaluación y Aprendizaje. En A. Benito y A. Cruz, (dirs.), Nuevas claves para la docencia universitaria en el Espacio Europeo de Educación Superior (pp. 87-100). Madrid: Narcea.

Cabero, J. (2004). Reflexiones sobre la brecha digital y la educación. En F. Soto y J. Rodríguez (coords.), Tecnología, Educación y Diversidad: Retos y realidades de la inclusión digital (pp. 23-42). Murcia: Consejería de Educación y Cultura.

Coll, C., Rochera, M.J. y Colomina, R. (2010). Usos situados de las TIC y mediación de la actividad conjunta en una secuencia instruccional de educación primaria. Electronic Journal of Research in Educational Psychology, 8, 517-540.

Delgado-García, A. M. y Oliver-Cuello, R. (2006). La promoción del uso del software libre por parte de las universidades. RED, Revista de Educación a Distancia, 17, 1-28. Consultado el 16/04/2009. En http://www.um.es/ead/red/17

García-Fernández, F. y Bringué Sala, X. (2002). Una familia en el ciberespacio. Cómo aprovechar Internet en la educación familiar. Madrid: Ediciones Palabra, S.A.

González Sanmamed, M. y Raposo Rivas, M. (2008). Necesidades formativas del profesorado Universitario en el contexto de la Convergencia europea. Revista de Investigación Educativa, 26, 285-306.

Fernández M. (2000). El Universo de las Nuevas Tecnologías: Información y Nuevas Tecnologías en la Enseñanza. Jornadas Internet en Educación. Netdidáctica, 5, 6-11.

Kaftan, J., Bucke, G.y Haack, A. (2006). Using Formative Assessments to Individualize. Instruction and Promote Learning. Middle School Journal, 37, 44-49.

Klenowski, V. (2005). Desarrollo de portafolios para el aprendizaje y la evaluación. Madrid: Narcea. 
Latiesa, M. (1996).Validez y fiabilidad de las observaciones sociológicas. En M. García, J. Ibáñez y F.Alvira. El análisis de la realidad social. Métodos y técnicas de investigación. Madrid: Alianza.

Lenhart, A., Rainie, L. y Lewis, O. (2001). Teenage life online: the rise of instantmessage generation and the internet's impact on friendship and family relatio hips. Washington, D.C.: Pew Internet \& American Life Project.

Losada, J. L. y López-Feal, R. (2003). Métodos de investigación en ciencias humanas y sociales. Madrid: Thomson.

Marquès, P. (2003). Funciones y limitaciones en las TIC en la educación. Universidad Autónoma de Barcelona. Consultado 6/09/2009. En: http://www.pangea.org/peremarques/siyedu.htm\#ventajas

McClintock, R. (1993). Elaboración de un nuevo sistema educativo. En R. McClintock, M. J. Striebel y G. Vazquez (Coords.), Comunicación, Tecnología y Diseños de Instrucción: La construcción del conocimiento escolar y el uso de los ordenadores (pp. 127-176). Madrid: CIDE-MEC.

Ministerio de Ciencia e Innovación. (2009). Datos básicos del sistema universitario español. Curso 2008/2009. Ministerio de Ciencia e Innovación. Secretaría de Estado de Universidades. Consejo de Coordinación Universitaria. 2008. Consultado el 20/02/2010. En: http://www.oei.es/salactsi/Informe2008-2009.pdf

Oblinger, D.y Oblinger, J. L. (2005). Educating the Net Generation.Washington, D.C.: Educause.

Ortega F. D. e Isla Montes J. L. (2000). Internet como medio tecnológico para el docente y el discente. Jornadas Internet en Educación. Netdidáctica, 5, 37-42.

Ruiz Corbella, M. y Quintanal Díaz, J. (2008). ¿Evoluciona la educación a distancia? Las Listas de distribución como protagonista De la innovación. El caso de la lista Cuedistancia (CUED-L). Revista de Investigación Educativa, 26, 323-346.

Ruiz, E. (2005). Evaluación de la calidad del aprendizaje de los alumnos en la titulación de Ciencias de la Actividad Física y del Deporte. Tesis doctoral no publicada. Universidad de León (León).

Salinas, J. (2004). Innovación docente y uso de las TIC en la enseñanza universitaria. Revista de Universidad y Sociedad del Conocimiento, 1(1). Consultado el 1/03/2009. En, http://www.uoc.edu/rusc/dt/esp/salinas1104.pdf 
Sigalés, C. (2004). Formación universitaria y TIC: nuevos usos y nuevos roles. Revista de Universidad y Sociedad del Conocimiento, 1(1). Consultado el 22/08/2009. En: http://www.uoc.edu/rusc/dt/esp/sigales0704.pdf

Suriá, R. (2006). Docencia, Diversidad y Discapacidad en el Alumnado. Electronic journal of research educational psychology, 4, 35-46.

Touriñan, J. M.; Rodríguez, A. y Olveira, E. (2003). La sociedad de la información y las nuevas exigencias en formación del profesorado. Aula Abierta, 81, 57-74.

Veen, W. y Vrakking, B. (2006). Homo Zappiens: growing up in a digital age. London: Continuum International Publishing Group.

Velázquez Buendía, R. y Martínez Gorroño, M.E. (2004). La autoevaluación y la coevaluación en el aprendizaje autónomo y cooperativo. En Hernández J. L. Álvarez y R. Velázquez (coords.), La evaluación en educación física. Investigación y práctica en el ámbito escolar (pp. 293-322). Barcelona: Graó. 


\section{Anexo}

Este cuestionario pretende obtener información sobre el conocimiento y uso de las nuevas tecnologías de la información y la comunicación (TIC). La información que nos proporciones será utilizada de una forma anónima. Muchas gracias por tu colaboración.

\begin{tabular}{|l|l|}
\hline Sexo & Mujer \\
\hline Hombre & \\
\hline Edad & \\
\hline
\end{tabular}

¿Cuál es su nacionalidad actual?

\begin{tabular}{|l|l|l|}
\hline España & Otro país de la Unión Europea & Latinoamérica \\
\hline
\end{tabular}

\begin{tabular}{|l|l|l|l|}
\hline \multicolumn{5}{|l|}{ Estado civil } \\
\hline Soltero & Casado & Separado & Viudo \\
\hline & & & \\
\hline
\end{tabular}

\begin{tabular}{|l|l|l|l|l|}
\hline \multicolumn{2}{|l}{ ¿Cuál es su situación laboral actual? } \\
\hline Trabajando & Desempleado & $\begin{array}{l}\text { Incapacidad labo- } \\
\text { ral }\end{array}$ & jubilado & Tareas del hogar \\
\hline & & & & \\
\hline
\end{tabular}

\begin{tabular}{|l|l|}
\hline Titulación que estudias & \\
\hline Curso en el que estás matriculado/a & \\
& \\
\hline
\end{tabular}

\section{NUEVAS TECNOLOGIAS}

\section{Accesibilidad y adaptabilidad}

Indica el grado en que tienes un acceso fácil a las nuevas tecnologías (TIC), es decir, el grado en el que su manejo está adaptado a tus posibilidades y capacidades.

\begin{tabular}{|l|l|l|l|l|l|}
\hline ¿Dispones de ordenador? & \multicolumn{2}{|l|}{ Si } & \multicolumn{2}{l|}{ No } \\
\hline ¿Tienes acceso a un ordenador? & Si & \multicolumn{2}{l|}{ No } \\
\hline ¿En qué lugar utilizas el ordenador? & En casa & $\begin{array}{l}\text { En el centro } \\
\text { de estudios }\end{array}$ & $\begin{array}{l}\text { En la } \\
\text { biblioteca }\end{array}$ & $\begin{array}{l}\text { En el } \\
\text { trabajo }\end{array}$ & $\begin{array}{l}\text { Otros } \\
\text { (señala } \\
\text { donde) }\end{array}$ \\
\hline ¿Tienes conexión a internet? & Si & & No \\
\hline
\end{tabular}




\section{Experiencia}

\begin{tabular}{|l|l|l|l|l|l|}
\hline ¿Sabes utilizar el ordenador? & Nada & Poco & Algo & Bastante & Mucho \\
\hline $\begin{array}{l}\text { ¿Cuál es tu nivel de conocimientos en el uso del } \\
\text { ordenador? }\end{array}$ & Nada & Poco & Algo & Bastante & Mucho \\
\hline ¿Sabes navegar por la red? & Nada & Poco & Algo & Bastante & Mucho \\
\hline $\begin{array}{l}\text { ¿Sueles acceder a internet? } \\
\text { ¿lectrónico, foros, acceder a páginas web) utili- } \\
\text { zas? }\end{array}$ & Nada & Poco & Algo & Bastante & Mucho \\
\hline ¿Cómo has aprendido a utilizarlos? & & foros & www & chats & mesenger \\
\hline
\end{tabular}

\section{Nuevas tecnologías y aplicación al grado}

\begin{tabular}{|l|l|l|l|l|l|l|}
\hline $\begin{array}{l}\text { ¿Crees que las nuevas tecnologías pueden ayudarte a } \\
\text { seguir a distancia tu carrera? }\end{array}$ & Nada & Poco & Algo & Bastante & Mucho \\
\hline $\begin{array}{l}\text { ¿Crees que es imprescindible saber manejar las } \\
\text { nuevas tecnologías para cursar tus estudios? }\end{array}$ & Nada & Poco & Algo & Bastante & Mucho \\
\hline $\begin{array}{l}\text { ¿En qué medida te sientes preparado para ayudarte } \\
\text { de las nuevas tecnologías en tu carrera? }\end{array}$ & Nada & Poco & Algo & Bastante & Mucho \\
\hline $\begin{array}{l}\text { ¿Crees que es positivo que se ofrezca la enseñanza } \\
\text { virtual frente a la presencial? }\end{array}$ & Nada & Poco & Algo & Bastante & Mucho \\
\hline $\begin{array}{l}\text { ¿En qué grado necesitas más formación en el manejo } \\
\text { de las nuevas tecnologías para cursar tus estudios? }\end{array}$ & Nada & Poco & Algo & Bastante & Mucho \\
\hline $\begin{array}{l}\text { ¿Estarías de acuerdo en recibir previa formación } \\
\text { sobre el uso de las TIC para cursar tu titulación? }\end{array}$ & Nada & Poco & Algo & Bastante & Mucho \\
\hline
\end{tabular}

